\title{
Optimization of a Water Window Capillary Discharge Radiation Source
}

\author{
M. Stefanovič, M. Vrbová
}

\begin{abstract}
Computer modeling of a fast electrical discharge in a nitrogen-filled alumina capillary was performed in order to discover discharge system parameters that lead to high radiation intensity in the so-called water window range of wavelengths $(2-4 \mathrm{~nm})$. The modeling was performed by means of the two-dimensional RMHD code $\mathrm{Z}^{*}$. The time and spatial distribution of plasma quantities were used for calculating the ion level populations and for estimating the absorption of the $2.88 \mathrm{~nm}$ radiation line in the capillary plasma, using the FLYCHK code. Optimum discharge parameters for the capillary discharge water window source are suggested. The heating of the electrodes and the role of capillary channel shielding were analyzed according to the $\mathrm{Z}^{*}$ code.
\end{abstract}

Keywords: water window radiation, capillary discharge, soft X rays, nitrogen plasma.

\section{Introduction}

Water window radiation sources are interesting for application in the biomedical sciences [1]. High absorption of this radiation by proteins, but very little absorption by oxygen, makes imaging of living cells possible.

We have looked for optimal parameters of the fast capillary discharge radiation source, namely capillary dimensions, electrodes' shapes and gas filling pressure, in order to achieve maximum radiation power in the water window range of wavelengths.

\section{RMHD computer modeling}

Calculations of plasma quantities were executed using the two-dimensional RMHD (radiative-magnetohydrodynamic) $Z^{*}$-code $[2,3]$. The physical model established in $\mathrm{Z}^{*}$ is based on the quasi-neutral multicharged ion plasma magneto-hydrodynamic equations with self-consistent electromagnetic fields and the radiation transport in a $2 \mathrm{D}$ axially symmetric geometry. This enables detailed engineering of the pinching discharge processes in the capillary. The $\mathrm{Z}^{*}$ computer code engine provides the radial and time evolutions of the plasma quantities, and also an estimation of the radiation generated in the capillary. These results are based on the input parameters inserted into the calculations by the user. These input

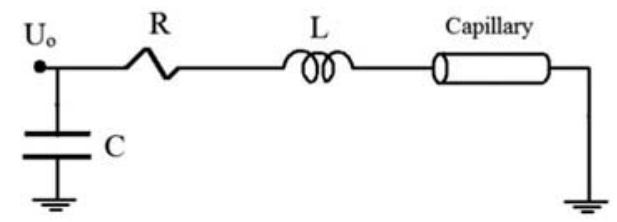

Fig. 1: Electrical scheme of the capillary discharge circuit parameters assume: external electrical circuit parameters, discharge geometry and properties of the filling gas (pressure, temperature, degree of pre-ionization, etc.).

\subsection{Code input}

\subsubsection{Electrical scheme of the discharge system}

The capillary discharge is a part of an under dumped RLC circuit (Figure 1). In accordance with the experimental setup [4], the following values are taken into account for the simulations: capacitor $C=15 \mathrm{nF}$ charged to initial voltage $U_{0}=80 \mathrm{kV}$, external resistivity and inductance of the circuit $R=0.73 \Omega$ and $L=50 \mathrm{nH}$, respectively. The capillary itself has its own impedance, which is incorporated into the code via the capillary geometry input.

\subsubsection{Capillary discharge geometry and filling gas}

Alumina $\left(\mathrm{Al}_{2} \mathrm{O}_{3}\right)$ capillary (magnetic permeability $\mu / \mu_{0}=8.5$, atomic weight $A=102$ ) filled with nitrogen gas $(Z=7, A=14)$ was investigated. Iron ( $Z=26, A=55.8$ ) electrodes were presumed.

Figure 2 shows the geometric details of the capillary grid used for the calculations. The capillary is presented in 2D cylindrical geometry. The left border of the drawing represents the axis of symmetry. The outer radius of the capillary is $21 \mathrm{~mm}$, while the inner radius $r$ is varied $(0.8,1.65$ and $2.5 \mathrm{~mm})$. The length of the capillary $l$ represents the distance between the two electrodes. The simulations were done in a range of capillary lengths $40-180 \mathrm{~mm}$. The capillary is closed at one end and opened at the other end. The open end electrode is indented $\sim 0.6 \mathrm{~mm}$ 
into the channel. The voltage supply electrodes are connected to the bottom and top right corner of the grid. Positive voltage is virtually applied to the electrode at the bottom.

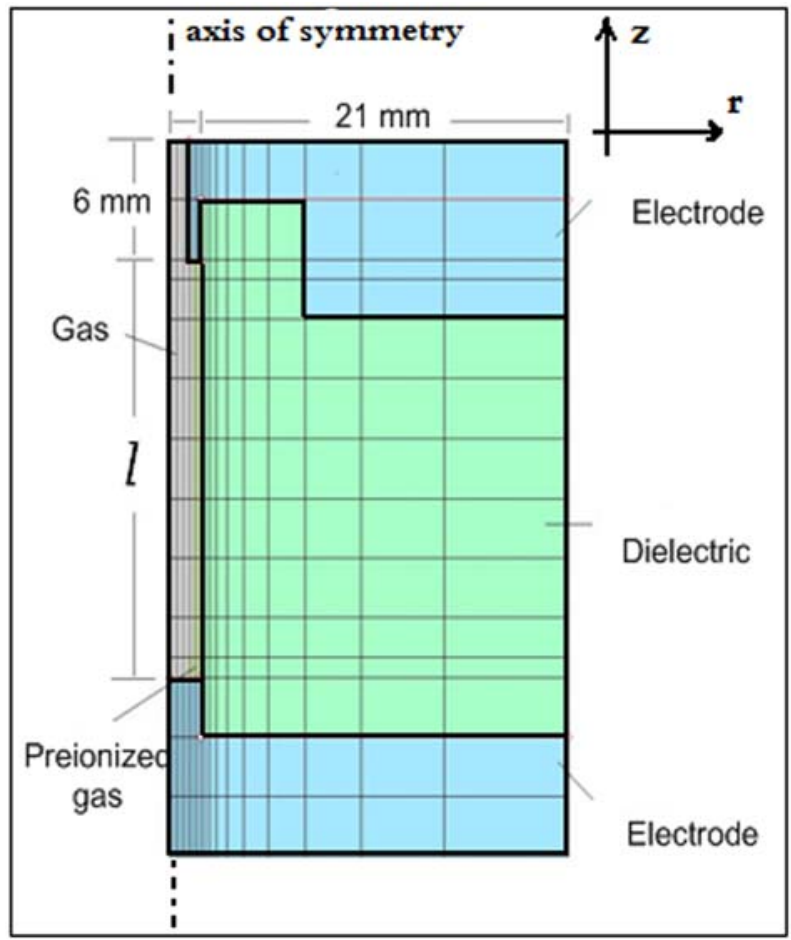

Fig. 2: Capillary grid in 2D cylindrical geometry

The nodes of the grid (Figure 2) correspond to the space coordinates at which the physical quantities are calculated. The grid is denser inside the plasma and around the plasma-electrodes and plasma-capillary boundaries. The thicknesses of the cathode and anode were chosen to be 9 and $6 \mathrm{~mm}$, respectively.

Nitrogen gas was chosen due to strong spectral lines in the wavelength region between 2 to $3 \mathrm{~nm}$. Also, a recent paper [5] confirmed nitrogen as a high water window emitter. The initial temperatures of the gas and the capillary wall are presumed $\sim 300 \mathrm{~K}$. A pre-ionized plasma channel with "effective preionization degree" $Z \sim 10^{-3}$ and electron temperature $\sim 0.05 \mathrm{eV}$ is presumed to be created in the gas cylinder near the alumina wall. Calculations were made for various initial molecular gas pressures in the range $13-667 \mathrm{~Pa}$.

\subsection{Results of modeling}

\subsubsection{Spatial and time distributions of plasma quantities}

When a charged capacitor is connected to the capillary channel, the electric current heats the plasma inside the channel, and its magnetic field causes plasma pinching. High temperature and dense plasma are created during the pinch. The plasma quantities depend substantially on the parameters of the discharge system, namely on the voltage applied, the discharge geometry and the filling gas. Figure 3 illustrates the distribution of the plasma electron density at time $t=85 \mathrm{~ns}$, shortly after the pinch. The electron density is uniform along the $z$ direction, and decreases monotonously in the radial direction. On the axis, the value of the electron density is around $10^{17} \mathrm{~cm}^{-3}$. The electron temperature is found to be homogeneous in a broad capillary volume $(0 \mathrm{~cm}<r<1 \mathrm{~mm})$ having a value of around $\mathrm{Te} \sim 50 \mathrm{eV}$ (Figure 4$)$.

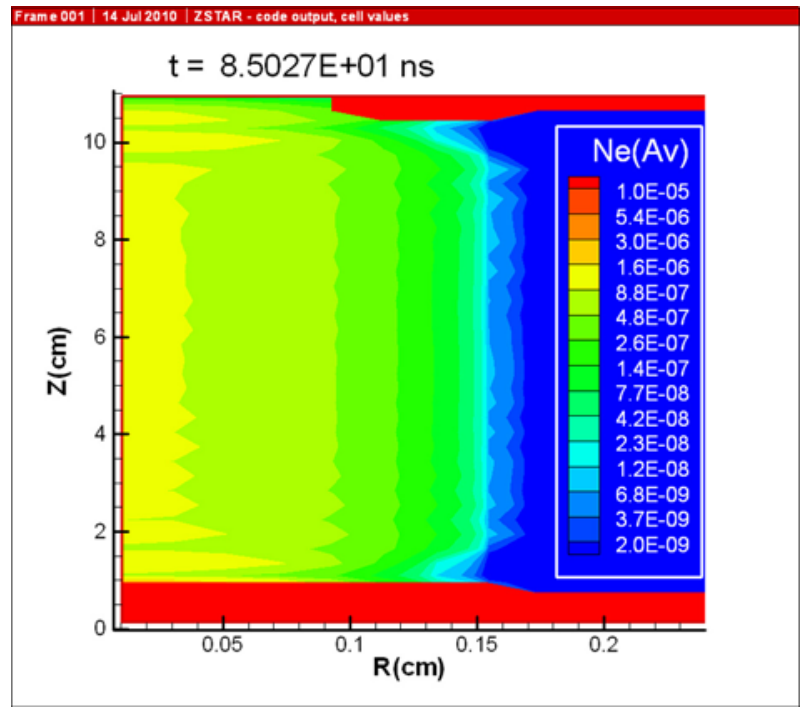

Fig. 3: Radial and longitudinal distribution of electron density $\mathrm{Ne}$ at time $t=85 \mathrm{~ns}$ (the units in the figure are given in Av; density expressed in $\mathrm{cm}^{-3}$ may be obtained by multiplying the value by the Avogadro constant $N a=6.02 \times 10^{23}$ )

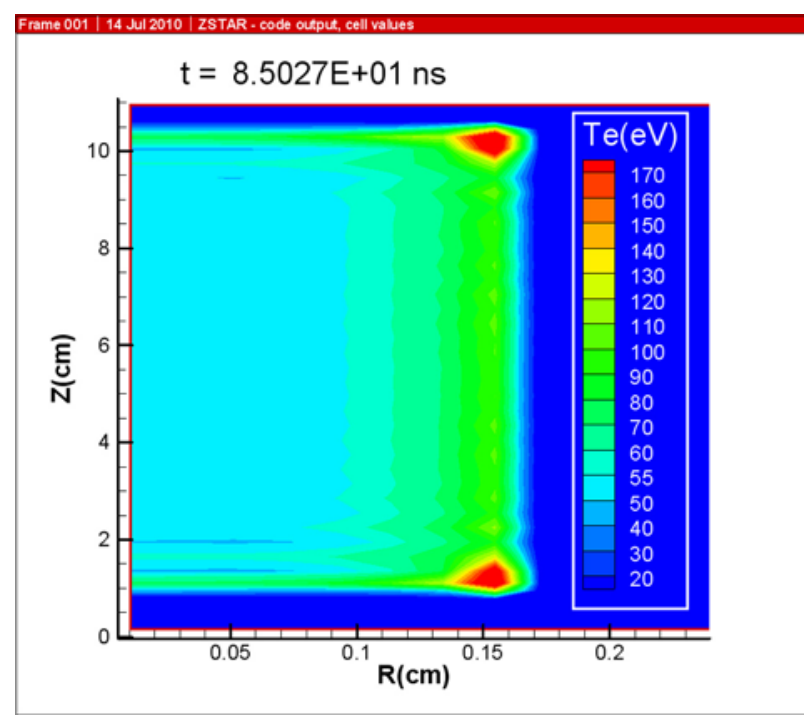

Fig. 4: Radial and longitudinal distribution of electron temperature $T e$ at time $t=85 \mathrm{~ns}$ 


\subsubsection{Output radiation estimated by the $\mathrm{Z}^{*}$ engine}

The code provides information on the output power of the radiation generated in the capillary, divided into 20 different spectral groups. As we are interested in radiation in the water window region, we observed the output emission power in group 13 of the $\mathrm{Z}^{*}$ code, corresponding to wavelength interval $2.1 \mathrm{~nm}<\lambda<3.1 \mathrm{~nm}$. We observed the evolution of the capillary plasma for the filling molecular gas pressure $p_{m}=80 \mathrm{~Pa}$. Figure 5 shows that the generated overall radiation (in the whole spectral range) $P_{\text {rad }}$ has two local maxima. The first radiation peak is reached at $\sim 15 \mathrm{~ns}$, and the second peak, which is much higher, appears at $t \sim 85$ ns. The second peak is related to the pinch. The radiation in spectral group 13 ( $\left.P_{\text {euv }}\right)$ has only one maximum at the pinch time. Its peak value is only 3 times lower than the peak value of total radiation $P_{\text {rad }}$. The radiation peaks come later than the current maximum.

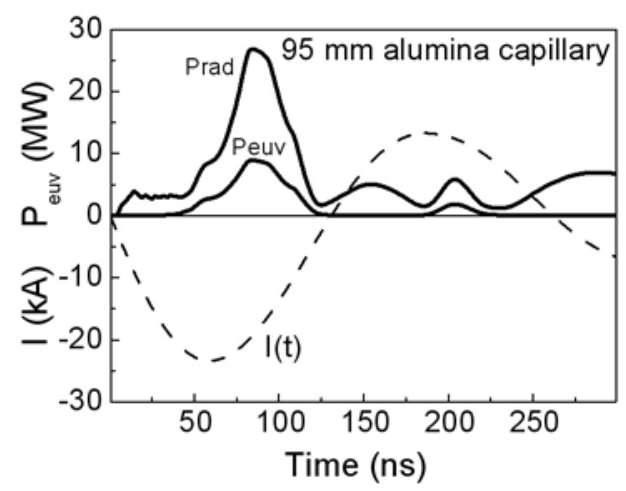

Fig. 5: Time dependences of evaluated electric current (dashed line), total radiation $P_{\text {rad }}$ and radiation $P_{\text {euv }}$ in the selected group 13

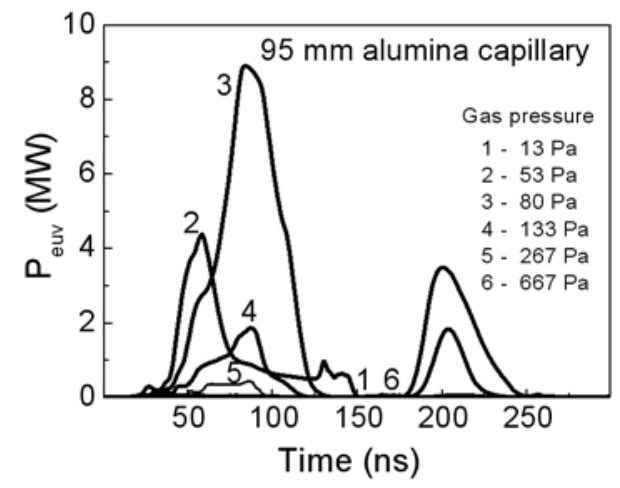

Fig. 6: Time dependencies of radiation power $P_{\text {euv }}$ in group 13 for various initial gas pressures

Also, simulations for various gas initial pressures in the range of $13-667 \mathrm{~Pa}$ were executed for a $1.65 \mathrm{~mm}$ capillary radius, in order to find the optimum filling gas pressure. The radiation outputs are very sensitive to changes in the initial gas pressure, as shown in Figure 6. The highest output radiation value is achieved for initial gas pressure $80 \mathrm{~Pa}$ at time $85 \mathrm{~ns}$. This is the optimum filling pressure of the gas for capillary radius $r=1.65 \mathrm{~mm}$. A capillary $l=95 \mathrm{~mm}$ in length is considered, but similar results in terms of optimum pressure are obtained for all capillary lengths $40-180 \mathrm{~mm}$.

The same procedure for finding the optimum gas pressure was repeated for two other capillary radii $(0.8$ and $2.5 \mathrm{~mm})$. The results are shown in Figure 7 . The line in Figure 7 shows the optimum pressure for the corresponding capillary radius. The optimum pressure is a monotonously decreasing function of the radius of the capillary.

If we now compare the optimized pressure output radiation powers for these three capillary radii, we can see that the highest emission is achieved for capillary radius $0.8 \mathrm{~mm}$, for filling gas pressure $400 \mathrm{~Pa}$ (Figure 8).

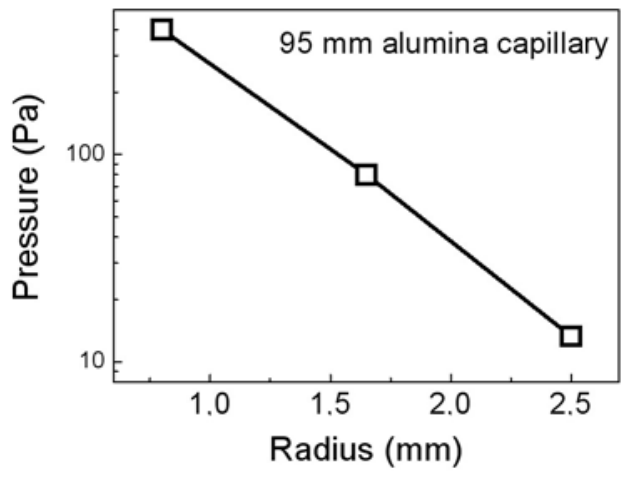

Fig. 7: Dependence of optimal pressure on the radius of the capillary

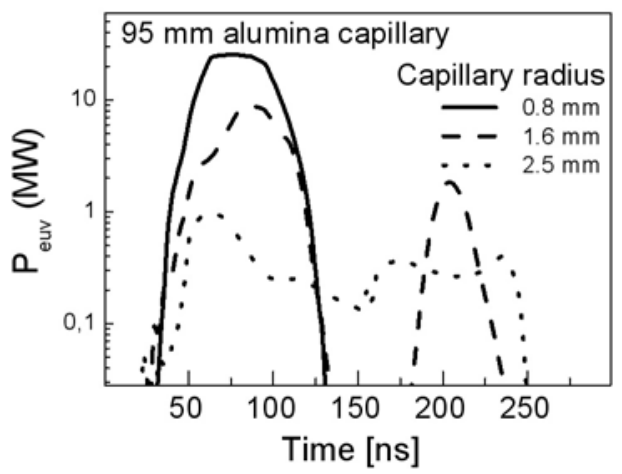

Fig. 8: Pressure optimized time dependences of radiation power $P_{\text {euv }}$ in group 13 for different capillary radii

\section{Spectrum according to FLYCHK}

The one-dimensional FLYCHK code [6] was used to estimate the spectral properties of the output radiation from the capillary. We considered the evolution 
of the plasma field quantities at the capillary centre $(r=0 \mathrm{~cm}, z=l / 2$, where $r$ and $z$ are radial and longitudinal coordinates, respectively, and $l$ is capillary length). Similar spectral properties of plasma are also expected in the cylinder along the capillary axis $0.3 \mathrm{~mm}$ in radius, because of the uniformity of the plasma quantities in this region (see Figures 3, 4).

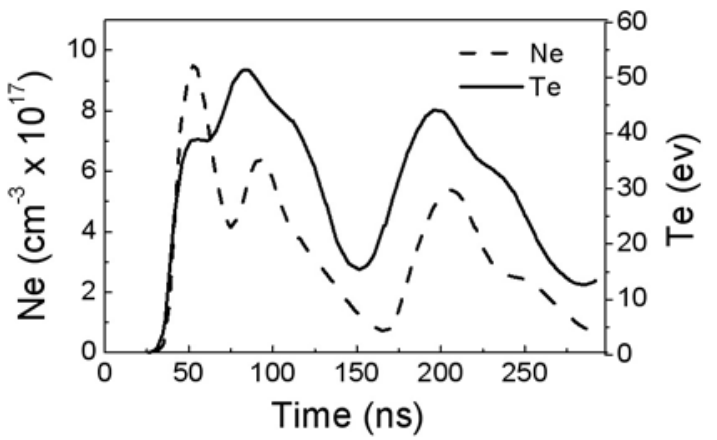

Fig. 9: Time evolutions of electron density and temperature calculated by $\mathrm{Z}^{*}$, for gas pressure $80 \mathrm{~Pa}$

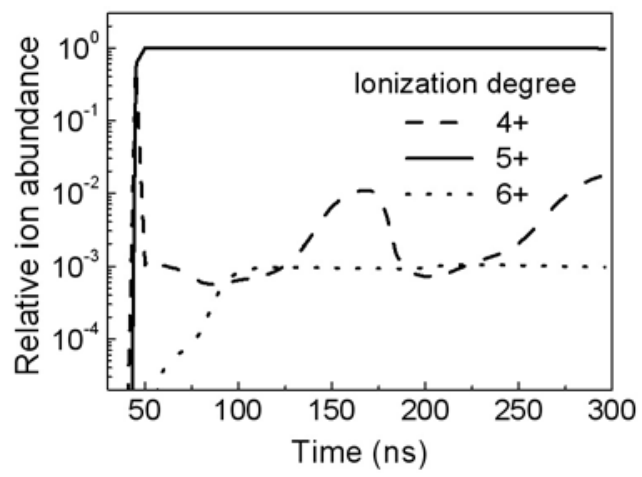

Fig. 10: Time dependences of relative nitrogen ion abundances for initial gas pressure $80 \mathrm{~Pa}$ (logarithmic scale)

The time evolutions of electron density $\mathrm{Ne}$ and electron temperature Te were taken from the $\mathrm{Z}^{*}$ code calculations (Figure 9), and were introduced as an input file into the FLYCHK code.

Non LTE plasma was presumed, and the simulations were executed in the time dynamic regime, because the electron density is not high enough to fulfill the McWhirter criterion [7] for the LTE plasma model. Optically thick plasma $5 \mathrm{~cm}$ in length is presumed. The resulting evolutions of the relative abundances of nitrogen ions for initial nitrogen pressure $80 \mathrm{~Pa}$ are shown in Figure 10 (only $4^{+}, 5^{+}$and $6^{+}$ ion fractions are shown, since other fractions have minor abundances). It is evident that He-like $N^{5+}$ ions prevail along the current period only after time $t \sim 50 \mathrm{~ns}$.

Instantaneous spectra at time $t=85 \mathrm{~ns}$ for $\lambda=2.1 .-3.1 \mathrm{~nm}$ are shown in Figure 11. We see that the strongest spectral line lying at the wavelength $\lambda=2.8785 \mathrm{~nm}$, corresponding to the quan- tum transition $1 \mathrm{~s}^{2}-1 \mathrm{~s} 2 \mathrm{p}$ in helium-like ions, prevails over the other lines. The optical depth $(\log (I o / I))$ of a $5 \mathrm{~cm}$ plasma column for different transitions is depicted in Figure 12. We see that there is huge absorption of the $2.88 \mathrm{~nm}$ spectral line in the plasma, i.e. plasma with such characteristics is almost totally nontransparent for this radiation. This conclusion is confirmed by applying the Elton formula to calculate the absorption coefficient [8]

$$
k=N_{l} \frac{g_{u}}{g_{l}} \cdot \frac{\lambda^{3}}{8 \pi c\left(\frac{\Delta \lambda}{\lambda}\right)} A_{u l}
$$

Where $\mathrm{k}$ is the absorption coefficient, $g_{u}$ and $g_{l}$ are statistical weights for the upper and lower atomic level, respectively, $N_{l}$ is the number of ions on the ground level, $\lambda$ is the radiation wavelength, $\Delta \lambda$ is the line width due to Doppler broadening, and $c$ is speed of light. Presuming $\Delta \lambda / \lambda=1.45 \times 10^{-4}$ [8], we calculated the value of the absorption coefficient $k=110 \mathrm{~cm}^{-1}$, which after multiplying by $5 \mathrm{~cm}$ length gives almost the same result as FLYCHK.

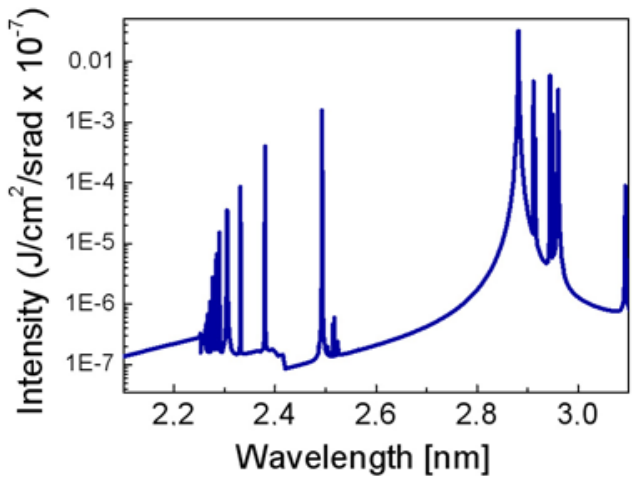

Fig. 11: Spectra for filling gas pressure $80 \mathrm{~Pa}$ at the time of maximum output radiation $t=85 \mathrm{~ns}$

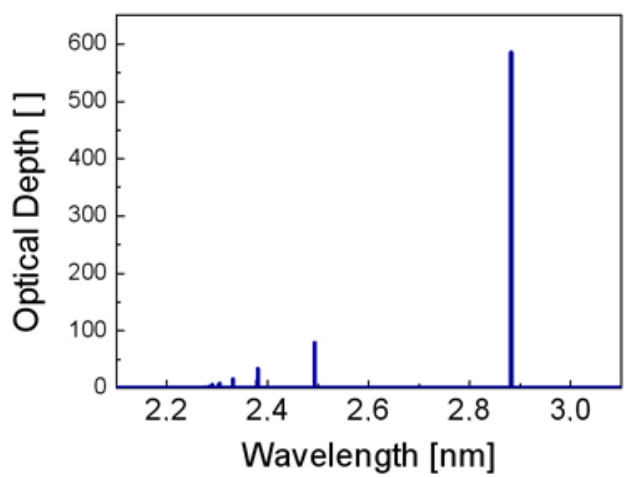

Fig. 12: Optical depth of the $5 \mathrm{~cm}$ plasma channel for gas pressure $80 \mathrm{~Pa}$ at the time of maximum output radiation $t=85 \mathrm{~ns}$

\section{Electrode heating}

A $\mathrm{Z}^{*}$ engine provides information on the temperature rise of electrodes $d T_{e l}$, from the beginning of the simulation. We investigated the heating of the electrodes 
during the discharge. Heating of the electrodes could be important when the source is working in a high repetition regime.

We considered the capillary discharge with the tip of one electrode protruded into the capillary channel (as in the experimental setup from reference [4] (Figure 13)), and compared it with the system with electrodes lying in the extension of the capillary wall and open at both ends (schematically presented in Figure 14). The protruded top is overheated accord-

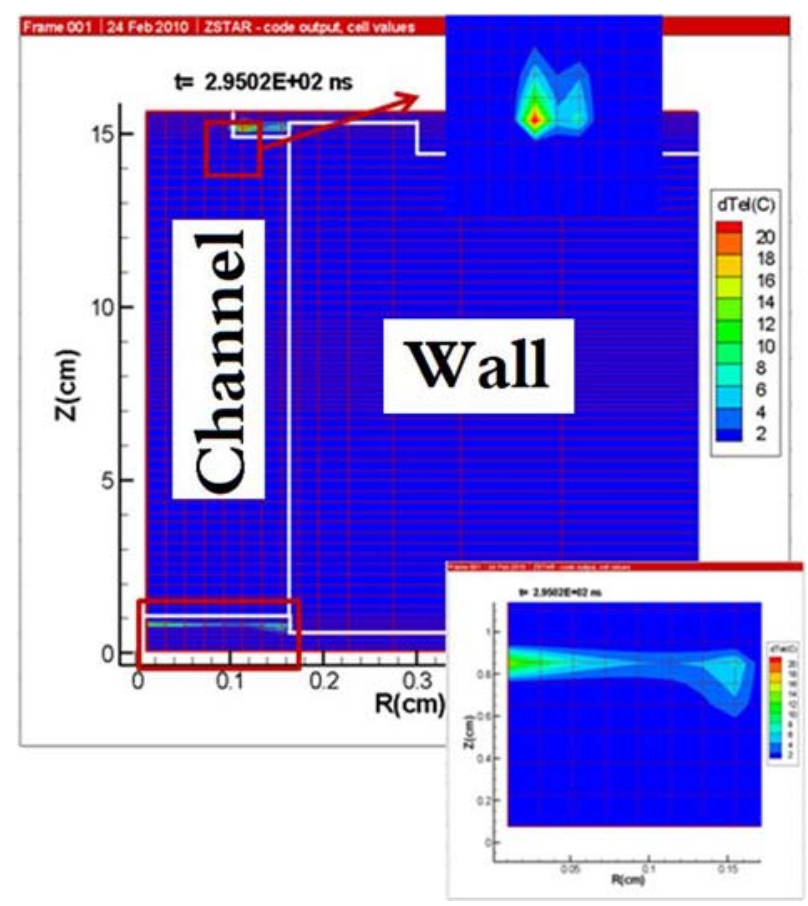

Fig. 13: Temperature increase of the electrodes for the discharge system with the tip drawn into the channel, after $300 \mathrm{~ns}$ from the beginning of the discharge; most heated parts are magnified

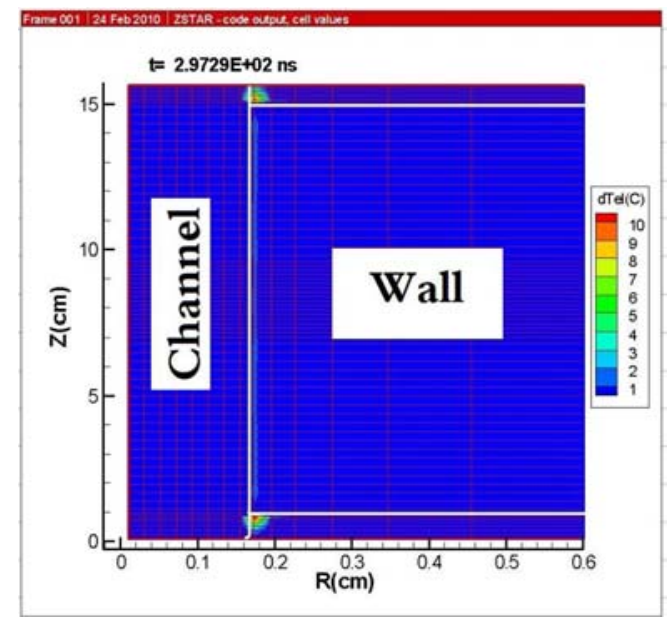

Fig. 14: Temperature increase of the electrodes for the discharge system with electrodes in the line with the capillary wall $300 \mathrm{~ns}$ after the beginning of the discharge ing to the $\mathrm{Z}^{*}$ code. On the other hand, the heating is two times lower for the discharge geometry in Figure 14. This leads to the conclusion that the tip of the electrode in the first scheme has an adverse effect on the heating of the electrodes.

\section{The role of capillary shielding}

In capillary discharge systems, the dielectric capillary is often surrounded by another metal in order to reduce the overall inductance in the discharge circuit. Namely, the smaller the inductance, the faster and higher the current pulse through the capillary will be. We performed simulations to investigate how discharge current and output radiation depend on the shielding of the capillary. For this purpose, special geometry of the capillary was inserted as an input into the $Z^{*}$ code (see Figure 15). The shield is $7 \mathrm{~mm}$ from the radial center of the capillary.

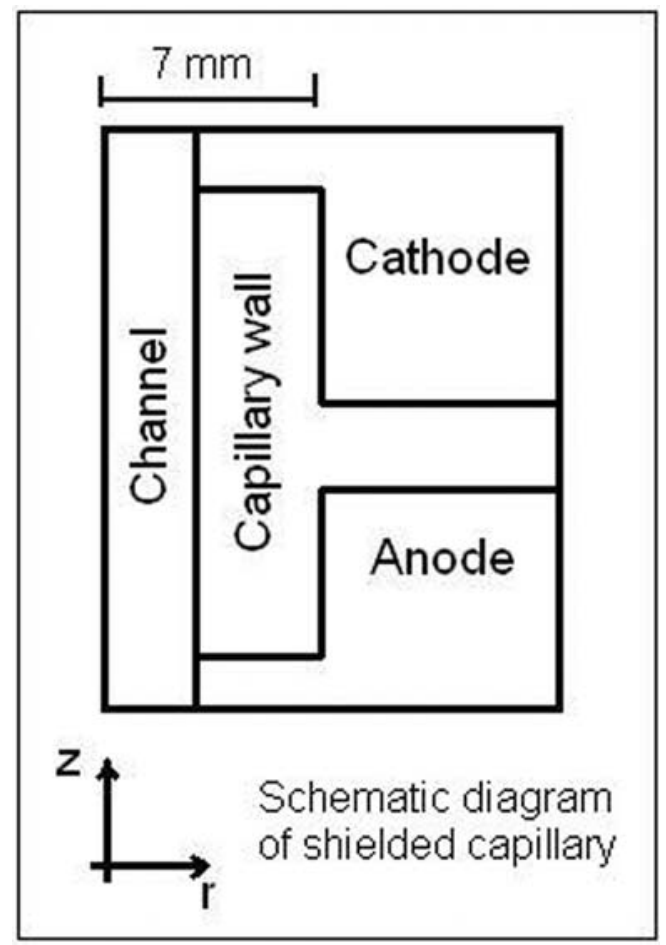

Fig. 15: Schematic diagram of a shielded capillary, represented in a cylindrically symmetrical geometry

The time dependences of the output radiation from the shielded and unshielded capillary, and also the corresponding discharge currents, are shown in Figure 16. The maximum output radiation in the $\mathrm{Z}^{*}$ 13 radiation group is approximately $15 \%$ higher for the shielded capillary. At the same time, the current slope is steeper and the maximum is higher.

We also calculated the distribution of the $z$ component of the electric field in the discharge sys- 


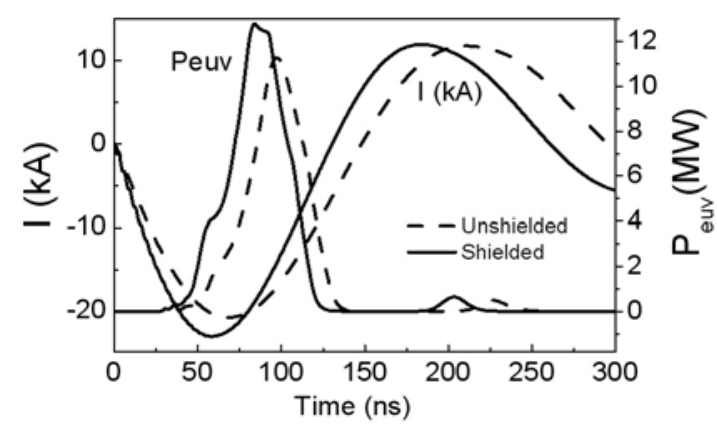

Fig. 16: Time dependences of the output radiation power in group 13 and the current for a shielded capillary (full line) and for an unshielded capillary (dashed line)

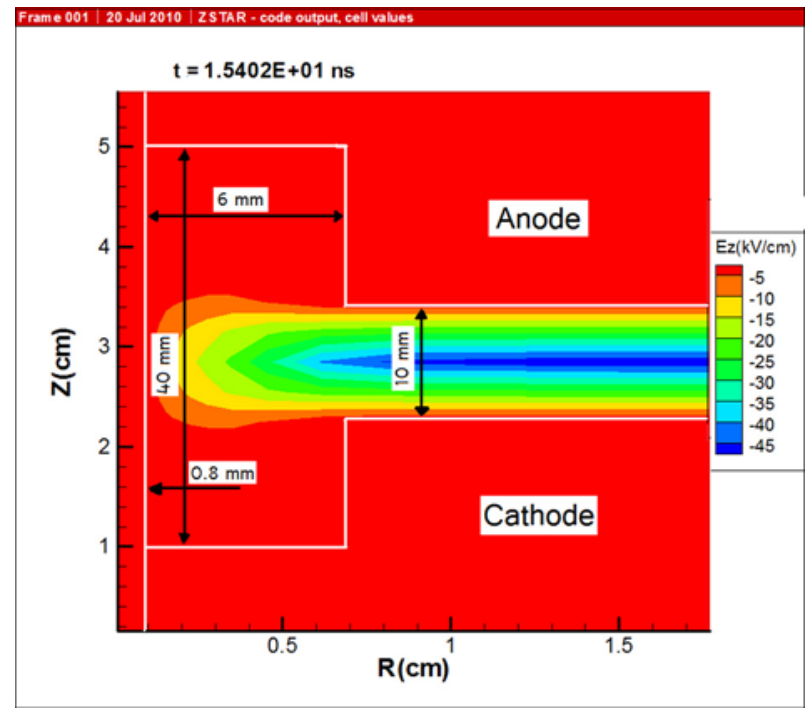

Fig. 17: Distribution of the z-component of the electric field in the discharge for the proposed geometry of the discharge (the axes are not proportional)

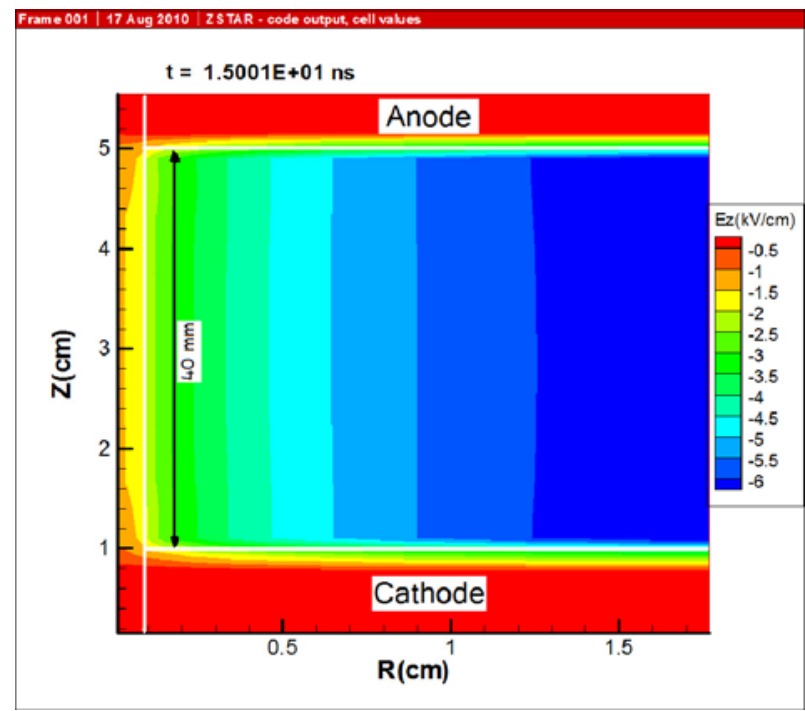

Fig. 18: Distribution of the $z$-component of the electric field in the discharge for the proposed geometry of the discharge (the axes are not proportional) tem at time 15 ns (the highest electric fields occur at this time, according to the $\mathrm{Z}^{*}$-code) for a shielded and unshielded capillary $4 \mathrm{~cm}$ in length (the radial component of the electric field is negligible in comparison with the $z$ component). The electrical field reaches a value of around $45 \mathrm{kV} / \mathrm{cm}$ in a shielded capillary (Figure 17), and only $6 \mathrm{kV} / \mathrm{cm}$ in an unshielded capillary (Figure 18). Since electrical breakdown in air occurs for an electric field of $30 \mathrm{kV} / \mathrm{cm}$, we conclude that electrodes must be isolated (either by sinking into dielectric oil or by separation using an insulator (i.e. alumina)) in a configuration with a shielded capillary.

\section{Suggested parameters of the new source}

We propose the parameters of a capillary discharge water window radiation source on the basis of the computer modeling described above. The electrical parameters of the discharge may be as follows: capacitor $C=15$, initial voltage $U_{0}=80 \mathrm{kV}$, external resistivity $R=0.73 \Omega$, and external inductance $L=50 \mathrm{nH}$.

Capillary radius $0.8 \mathrm{~mm}$ is most suitable for a source with gas pressure of $400 \mathrm{~Pa}$. The capillary channel length should be only $4 \mathrm{~cm}$, due to the high absorption of the water window radiation in the plasma column. Due to the high electric fields in a system with a shielded capillary, we suggest that an unshielded capillary be used as the source. The suggested discharge geometry is shown in Figure 18.

The time dependences of the current and radiation power in the $Z^{*} 13$ radiation group for the proposed system are shown in Figure 19. The highest XUV emission occurs approximately at the time of the current maximum, which is around $50 \mathrm{~ns}$.

The electron density and temperature distributions at the time of the emission peak are shown in Figures 20 and 21. The electron density is uniform along the $\mathrm{z}$ direction, while it decreases monotonously in the radial direction. The electron temperature is homogeneous in a broad capillary volume, with a value around $70 \mathrm{eV}$, suitable for creating $N^{5+}$ ions.

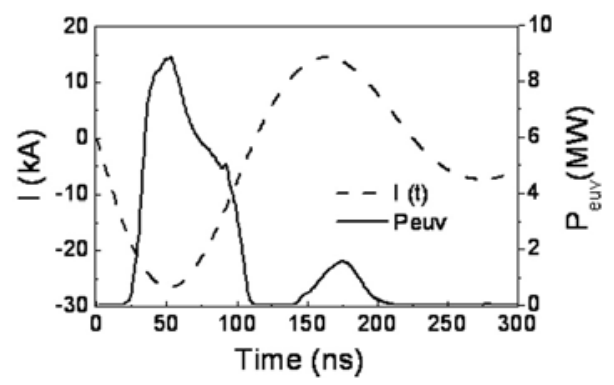

Fig. 19: Time dependences of the output radiation power in group 13 and the current for the proposed geometry 


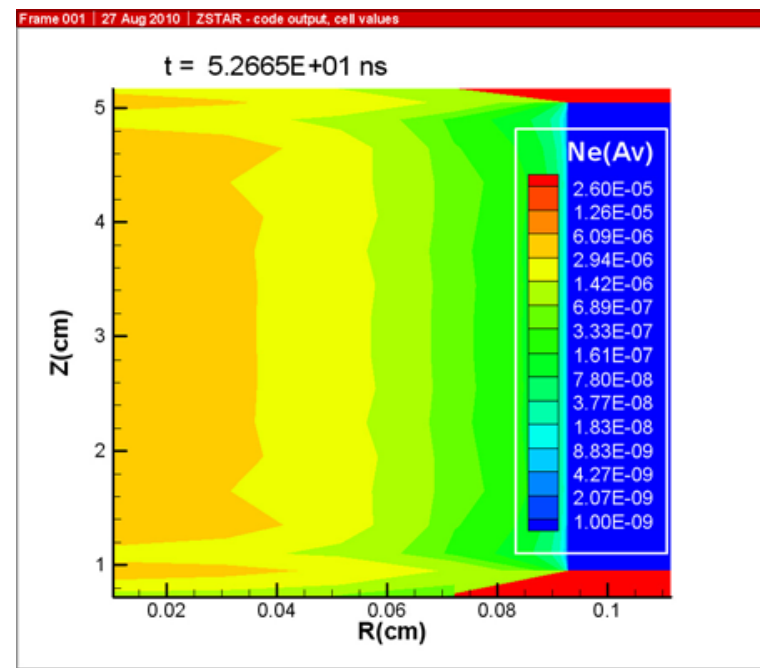

Fig. 20: Radial and longitudinal distribution of electron density $\mathrm{Ne}$ at the time of maximum current (the units in the figure are given in Av; the density expressed in $\mathrm{cm}^{-3}$ can be obtained by multiplying the value by the Avogadro constant $N a=6.02 \times 10^{23}$ )

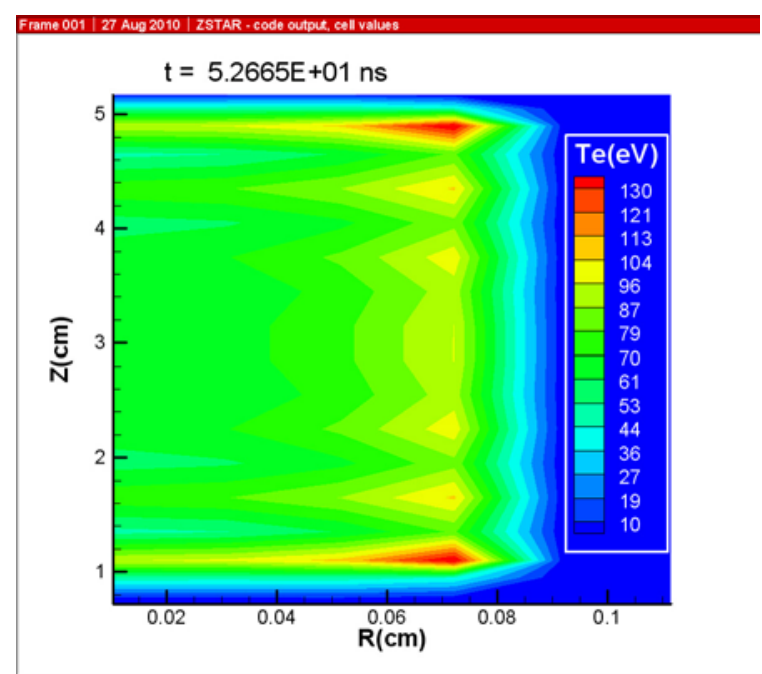

Fig. 21: Radial and longitudinal distribution of electron temperature $T_{e}$ at the time of a current maximum

\section{Conclusions}

The RHMD $\mathrm{Z}^{*}$ code was used to model a capillary pinching discharge to get an incoherent and polychromatic "water window" radiation source. The radiation outputs in group $13(2.1-3.1 \mathrm{~nm})$ were evaluated for different initial gas pressure, different capillary radii and different capillary lengths. The optimal radius of the system was proposed $(0.8 \mathrm{~mm})$, with filling gas pressure around $400 \mathrm{~Pa}$ and capillary length $40 \mathrm{~mm}$. Using the FLYCHK code as a postprocessor, the detailed kinetics of nitrogen ions were computed and the relative abundances of nitrogen ions were evaluated. After $50 \mathrm{~ns}, N^{5+}$ ions prevail over the other ions throughout the current period. FLYCHK simulations showed very high absorption of the $2.88 \mathrm{~nm}$ radiation line in the plasma. Electrode heating was investigated for two different discharge configurations. The role of capillary shielding was analyzed by $\mathrm{Z}^{*}$.

\section{Acknowledgement}

The authors gratefuly acknowledge professor Sergey Zakharov for his useful advice on the $\mathrm{Z}^{*}$ code and on pinching discharges. This research was supported by MEYS Research Project C42 and by MEYS Research Project INGO LA 08024.

\section{References}

[1] Ford, T. W.: Imaging Cells Using Soft X ray. In: From Cells to Proteins: Imaging Nature across Dimensions, Amsterdam : Springer Netherlands, 2005, p. 167-185.

[2] Zakharov, S. V., Novikov, V. G., Choi, P.: Z*code for DPP and LPP source modeling. In: EUV Source for Lithography. (Ed. V. Bakshi). Bellingham, Washington : SPIE PRESS, 2005, p. 223-275.

[3] Zakharov, S. V., Novikov, G. V., Mond, M., Choi, P.: Plasma Dynamics in Hollow Cathode Triggered Discharge with Influence of Fast Electrons on Ionization Phenomena and EUV Emission. Plasma Sources Sci. Technol. 17 (2), 2008, p. 13.

[4] Nevrkla, M.: Návrh a realizace zař́izení pro studium kapilárniho výboje v argonu: Diplomová práce. Praha : ČVUT - Fakulta jaderná a fyzikálně inženýrská, 2008.

[5] Vrba, et al.: Capillary pinching discharge as water window radiation source. 29th ICPIG, Cancún, México, 2009.

[6] Lee, R. W., Larsen, J. T.: A time-dependent model for plasma spectroscopy of K-shell emitters, JQSRT 56, 1996, p. 535-556.

[7] McWhirter, R. W. P.: In: Plasma Diagnostic Techniques? (Eds. R. H. Huddlestone, S. L. Leonard), Academic Press, New York, 1965, p. 201-264.

[8] Elton, C. R.: X ray lasers. London : Academic Press, 1990.

Ing. Miloš Stefanovič

Prof. Miroslava Vrbová, CSc.

E-mail: stefanovic@fbmi.cvut.cz

Department of Natural Sciences

Faculty of Biomedical Engineering

Czech Technical University in Prague

nám. Sítná 3105, Kladno 2, Czech Republic 